\title{
ESTUDIO DE LA DIVERSIDADY TAXONOMÍA DE HONGOS COMO ESTRATEGIA PARA LA ENSEÑANZA DE LA BIOLOGÍA
}

\section{Study of Diversity and Taxonomy of Fungi as a Strategy for the Teaching of Biology}

\section{Estudo da diversidade e taxonomia dos fungos como estratégia para o ensino da biologia}

Hugo Mauricio Jiménez M. ${ }^{1}$

Fecha de recepción: 14 de octubre de 2017

Fecha de aceptación: 10 de abril de 2018

\section{Resumen}

Este artículo describe cómo ha sido la enseñanza del curso de Biología de Hongos del Departamento de Biología de la Universidad Pedagógica Nacional, la cual se ha basado en el estudio integral, académico e investigativo de la diversidad y taxonomía de hongos, y la aplicación de estrategias como prácticas de laboratorio con microhongos y macrohongos, seminarios y proyectos cortos de investigación, que logran un aprendizaje significativo en los estudiantes de la Licenciatura en Biología con amplios conocimientos en morfología, fisiología, diversidad, ecología, taxonomía, y aplicaciones ambientales y biotecnológicas de hongos. Este artículo también presenta una breve revisión taxonómica sobre el Reino de los Hongos que incluye sus características morfológicas y ecológicas.

Palabras clave: diversidad de hongos; taxonomía de hongos; estrategias pedagógica; microhongos; macrohongos; enseñanza de los hongos

1 Profesor Catedrático Departamento de Biología, Universidad Pedagógica Nacional (Colombia). 


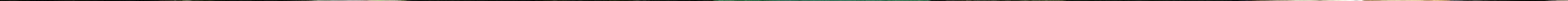




\section{Abstract}

This paper describes how the teaching of Fungal Biology course of the Biology Department at National Pedagogical University has been, this has been based on the integral, academic and investigative study of diversity and taxonomy of fungi, and the application of strategies such as laboratory practices with micro-fungi and macro-fungi, seminars, and short research projects, which achieve a significant learning experience for students of the Bachelor's degree of Biology with extensive knowledge in morphology, physiology, diversity, ecology, taxonomy, and environmental and biotechnological applications of fungi. This paper also presents a brief taxonomic review on the Kingdom of Fungi including its morphological and ecological characteristics.

Key Words: diversity of fungi; fungal taxonomy; pedagogical strategies; microfungi; macromycetes; teaching fungi

\section{Resumo}

Este artigo descreve como foi ensinar o curso de Biologia Fúngica do Departamento de Biologia da Universidade Pedagógica Nacional, que é baseado no estudo abrangente, acadêmico e de pesquisa da diversidade e da taxonomia dos fungos, e a aplicação de estratégias como práticas de laboratório com micro-fungos e macrofungos, seminários e projetos curtos de pesquisa que atingem a aprendizagem significativa em alunos da Licenciatura em Biologia com amplo conhecimento na morfologia, fisiologia, diversidade, ecologia, taxonomia e aplicações ambientais e biotecnológicas dos fungos. Este artigo também apresenta uma breve revisão taxonômica sobre o Reino dos Fungos, que inclui suas características morfológicas e ecológicas.

Palavras-chave: diversidade de fungos; taxonomia fúngica; estratégias pedagógicas; microfungos; macrofungos; ensino dos fungos

La construcción del conocimiento científico y la enseñanza de la ciencia hacen parte de un proceso de construcción social, que busca la adquisición de capacidades conceptuales, procedimentales y actitudinales en los estudiantes; con el fin de formarlos como ciudadanos con conocimiento científico [...]. Para lograrlo el proceso de enseñanza-aprendizaje debe estar mediado por una serie de estrategias, que les permita a los estudiantes aprender conceptos y construir modelos, desarrollar destrezas cognitivas, experimentales [...]. (Angarita, 2011, p.16).

La enseñanza de las ciencias favorece en los educandos el desarrollo de sus capacidades de observación, análisis, razonamiento, comunicación y abstracción; y a su vez permite que elaboren su pensamiento de manera autónoma.

En la enseñanza de la biología, "la observación y la acumulación de conocimientos son indispensables para la generación de hipótesis o teorías", y así mismo "la generación del conocimiento científico requiere de una interacción entre tres grandes componentes: la observación (el método inductivo), el análisis del marco conceptual teórico, y la experimentación (el método hipotético deductivo). (Balvanera, 1995, pp. 3, 5).

“La observación y la experimentación son parte inherente de la generación de conocimiento [en ciencias biológicas], y por lo tanto deben ser incorporadas en la enseñanza de las ciencias naturales". "Es indudable que la planeación de observaciones o experimentación con sistemas biológicos sencillos paralelos a la exposición de la teoría requiere de mucho trabajo por parte del maestro" (Balvanera, 1995, p.7).

El diseño y la realización creativa de variadas actividades para la enseñanza de las ciencias biológicas son fundamentales, y en especial para la enseñanza de la biología de hongos, pues estos ocupan el segundo lugar en biodiversidad en el planeta después de los insectos, y en un país tan biodiverso como Colombia los hongos serían de gran interés; por otra parte, una manera de enseñar ciencias biológicas es mediante el estudio de diversidad biológica.

En el planeta hay una gran biodiversidad; se estiman unos 15 millones de especies y se han clasificado taxonómicamente en los principales grupos: virus, arqueobacterias, bacterias, hongos, protozoos, algas, plantas, nematodos, moluscos, crustáceos, insectos, arácnidos, aves, anfibios, reptiles, peces y mamíferos. La diversidad de hongos se estima en 1.5 millones de especies; por su alta variabilidad, su clasificación taxonómica se actualiza constantemente y se corrobora con los avances en Filogenia Fúngica y Sistemática Molecular Fúngica.

En los últimos 20 años, he tenido la oportunidad de estudiar la diversidad de microhongos y macrohongos de bosques altoandinos de Colombia y desde el 2012 he 
sido maestro de la asignatura de Biología de Hongos en el Departamento de Biología de la Universidad Pedagógica Nacional. Allí he adelantado un trabajo de contextualización sobre microhongos y macrohongos; estudiados mediante su morfología, fisiología, diversidad, ecología, taxonomía, y aplicaciones ambientales y biotecnológicas con el fin de viabilizar procesos de aprendizaje en los estudiantes de Licenciatura en Biología; que los lleve a una formación integral en relación con lo académico, investigativo y pedagógico (Jiménez, 2018).

A continuación presento una breve descripción taxonómica de hongos a partir de David Hibbett (2007) quien junto con 67 investigadores a nivel mundial (13 países) publicaron en Mycological Research un artículo en donde actualizaron la taxonomía del Reino de los Hongos y se establecieron 7 Phyla: Microsporidia, Blastocladiomycota, Neocallimastigomycota, Chytridiomycota, Glomeromycota, Ascomycota y Basidiomycota, esta clasificación se basó en estudios de Sistemática Molecular Fúngica a partir de genes múltiples de rRNA.

En el Phylum Microsporidia se encuentran hongos unicelulares que son parásitos intracelulares obligados de animales vertebrados, artrópodos, moluscos, anélidos, nematodos y protistas como los ciliados (Hibbett et al., 2007; Keeling y Fast, 2002).

Los Phyla Blastocladiomycota y Neocallimastigomycota antes del 2007 eran órdenes del Phylum Chytridiomycota, pero por los estudios moleculares se establecieron como Phyla separados; estos dos Phyla, a su vez, se diferencian por la morfología de sus zoosporas, además los microhongos del Phylum Neocallimastigomycota son anaeróbicos y se encuentran de manera primordial en el rumen de herbívoros (Blackwell et al., 2007; Hibbett et al., 2007).

Los microhongos del Phylum Chytridiomycota se encuentran en agua dulce como lagos, lagunas y ríos, se caracterizan por hifas hialinas aseptadas de 8-10 $\mu \mathrm{m}$ de ancho, micelio cenocítico hialino y pared celular compuesta por quitina y glucano; en su fase asexual producen un zoosporangio con zoosporas que tienen flagelo en forma de látigo. Sus clases son Chytridiomycetes y Monoblepharidomycetes (Hibbett et al., 2007).

El curso de Biología de Hongos lo diseñé para que los estudiantes conozcan la diversidad de hongos a partir del estudio de su taxonomía actual y con la observación de microhongos del Phylum Glomeromycota y Ascomycota, y de macrohongos del Phylum Basidiomycota.
Los microhongos del Phylum Glomeromycota están en el suelo principalmente con hifas aseptadas de 8-10 $\mu \mathrm{m}$ de ancho, micelio cenocítico, pared celular compuesta por quitina y quitosan, su clase Glomeromycetes abarca las endomycorrizas que son simbiontes intracelulares de plantas y forman estructuras como apresorio, arbúsculo, vesícula y esporas externas para su reproducción, su Subphylum Mucoromycotina incluye a los hongos de formación de esporangiosporas a partir de un esporangio originado en un espongióforo cuyos representantes más conocidos son Mucor sp., Rhizopus sp., Zygorrhynchus sp. y Absidia sp.

Los hongos del Phylum Ascomycota son un grupo muy diverso, se encuentran en variados ambientes como suelo, agua salada, agua dulce y en todos los climas, presentan aplicaciones ecológicas como saprófitos y patógenos de plantas y animales, presentan hifas septadas en promedio de $2.5 \mu \mathrm{m}$ de ancho, micelio septado y pared celular compuesta por quitina y glucano. Sus estructuras reproductivas son el parámetro para su identificación, presentan una fase sexual conocida como telomorfo por la formación de ascosporas dentro de un asco, que a su vez forma unas estructuras reproductivas conocidas como peritecios de la clase Sordariomycetes, cleistotecios de la clase Eurotiomycetes y apotecios de la clase Pezizomycetes; y una fase asexual conocida como anamorfo por la formación de conidios, los cuales pertenecen a la Forma-Taxón: Deuteromycetes, y dependiendo de sus estructuras reproductivas y del origen de los conidios, ya sea enteroblástico o fialídico, la forma, el tamaño y el color, se pueden identificar estos microhongos inclusive a especie.

Los macrohongos del Phylum Basidiomycota presentan hifas septadas de $2.5 \mu \mathrm{m}$ de ancho en promedio, micelio septado, presencia de septos dolíporos y fíbulas que permiten su condición dicarionte, además, pared celular compuesta por quitina y glucano. Sus cuerpos fructíferos son el parámetro para su identificación: en su fase sexual presentan la formación de basidios que dan origen a basidiosporas, los cuales se encuentran en el himenio, este puede ser en forma de lamelas que están en el píleo para el caso del orden Agaricales o himenio poroso en la zona ventral para el caso del orden Polyporales, estos dos órdenes se encuentran en la clase Agaricomycetes.

Los Agaricomycetes se observan en los bosques, principalmente en épocas húmedas, y cumplen varias funciones ecológicas, como el ser parásitos de árboles, saprofitos, que se encuentran en troncos en descomposición, ramas y hojarasca; o simbiontes, como es el caso de las ectomycorrizas, en donde las hifas de los hongos tienen una relación intercelular con las raíces de las plantas y forman la red de Hartig. 
En el curso electivo de Biología de Hongos del Departamento de Biología de la Universidad Pedagógica Nacional para estudiantes de Licenciatura en Biología de fundamentación y profundización, he diseñado una práctica de laboratorio para que aprecien y conozcan la diversidad de microhongos. Esta práctica consiste en el aislamiento de microhongos del aire en el campus de la universidad, para ello, colocamos una caja de petri destapada por 15 minutos con el medio de cultivo Papa Dextrosa Agar debajo de árboles de gran tamaño debido a que la flora microbiana es alta en estas zonas, luego tapamos la caja de petri y la dejamos incubando por 7 días a temperatura ambiente. Después realizamos conteo de Unidades Formadoras de Colonia (UFC) y describimos las colonias de los microhongos ya sean algodonosas, aterciopeladas o polvorientas; a continuación, para cada colonia hacemos montajes con láminas, azul de Lactofenol y cinta pegante transparente, y observamos al microscopio e identificamos los microhongos según Género y en algunos casos a Especie.

Con esta práctica los estudiantes adquieren habilidades y destrezas en la descripción de las colonias e identificación de microhongos. También aprenden una metodología que es fácil de realizar e integran la teoría con la práctica.

En estas prácticas hemos identificado los microhongos del Phylum Glomeromycota: Mucor sp., Rhizopus sp., Zygorrhynchus sp. y Absidia sp.; y del Phylum Ascomycota: Aspergillus fumigatus, Alternaria sp., Cladosporium herbarum, Cladosporium cladosporoides, Fusarium sp., Drechslera sp., Penicillium sp., Trichoderma harzianum, Trichoderma sp., Trichotecium sp., entre otros.

Otra práctica de laboratorio en la que los estudiantes conocen la diversidad de macrohongos es a partir de la realización de dibujos descriptivos de ejemplares del orden Agaricales y Polyporales de la Colección de Macrohongos del Departamento de Biología - UPN, los estudiantes han acogido positivamente esta práctica, ya que a partir de ejemplares identificados aprenden sus nombres científicos y también consultan su clasificación taxonómica actualizada, además adquieren experiencias en la observación de macrohongos, que podrán ser aplicadas en sus salidas de campo.

Los macrohongos de estudio son: Amanita muscaria, Amanita sp., Agaricus sp., Auricularia sp., Boletus sp., Boletus edulis, Boletus luteus, Bovista sp., Clavicorona pyxidata, Coltricia sp., Coriolus sp., Corticium sp., Coprinus sp., Cyathus sp., Fomes sp., Galerina sp., Ganoderma sp., Gymnopilus sp., Hydnopolyporus sp., Laccaria sp., Lentinellus sp., Lepiota rhacodes, Lepiota sp., Lycoperdon sp., Lycoperdon perlatum, Macrolepiota sp., Marasmius sp., Paxillus sp., Psathyrella sp., Pycnoporus sanguineus, Schizophylum sp., Serpula sp., Stereum sp. y Trametes sp.
También realizo una actividad en la que los estudiantes diseñan una Fotogalería de macrohongos con fotos que han adquirido en salidas de campo en el transcurso de su licenciatura, con mi apoyo los identificamos a Genero y los estudiantes consultan su clasificación taxonómica actualizada y las aplicaciones ecológicas como ectomycorrizas, saprofitos o parásitos.

En estas actividades prácticas de laboratorio con microhongos, macrohongos y Fotogalería he fomentado que los estudiantes trabajen en grupos, lo cual ha sido muy enriquecedor y como lo analizan Pantoja y Covarrubias (2013), al participar en equipos los estudiantes se motivan y mejoran sus habilidades de participación, integración y transferencia de conocimientos. Cuando se fomentan estos tipos de trabajo cooperativo se genera interés y compromiso por parte de los estudiantes, y así mismo los integrantes incorporan mecanismos críticos de retroalimentación y comparación con otras formas de pensar.

Otra estrategia de aprendizaje que he diseñado para que los estudiantes conozcan y aprecien la diversidad y taxonomía de microhongos y macrohongos es la realización de seminarios, los estudiantes escogen un Género de un hongo, realizan un escrito y exponen a sus compañeros los resultados de la consulta sobre su clasificación taxonómica actual, la estructura reproductiva que permite su identificación, descripción y aplicaciones ecológicas, ambientales o biotecnológicas.

En investigación formativa motivo a los estudiantes para que diseñen y desarrollen un proyecto corto de investigación sobre diversidad ya sea de microhongos o macrohongos; en este proyecto los estudiantes adquieren habilidades investigativas y más aprendizajes sobre hongos, de igual manera, aprenden cómo escribir un artículo científico y cómo exponerlo a sus compañeros, experiencias que les servirán cuando realicen un trabajo investigativo y/o trabajo de grado (Jiménez, 2012).

Desde el 2012 como maestro de Biología de Hongos en el Departamento de Biología - UPN y con todas estas estrategias didácticas para la enseñanza de la biología, he notado un gran gusto por la asignatura y enriquecimiento académico por parte de los estudiantes, como lo demuestran algunos de sus comentarios (Véase tabla 1), ya que han tenido un aprendizaje integral e investigativo sobre la diversidad y taxonomía de hongos, y para mí ha sido un gran logro profesional el poder transmitir mis conocimientos y experiencias en hongos a los estudiantes de Licenciatura en Biología de la Universidad Pedagógica Nacional. 
En la tabla 1 muestro algunos comentarios de los estudiantes en relación con el curso electivo de Biología de Hongos desde el 2012.

\section{Tabla 1}

Estudiante 1. 2012-2. La estrategia de enseñanza del curso es buena y pertinente porque se integran fundamentos teóricos con las prácticas de laboratorio, y estas incentivan el desarrollo experimental.

Estudiante 2. 2013-1. Se logra aprender la identificación de microhongos y macrohongos, y el proyecto de investigación permite reconocer la riqueza de hongos de distintos lugares de Colombia.

Estudiante 3. 2014-2. El curso me ayudó a entender las características y la importancia de las diferentes taxas de los hongos, ya que son cosmopolitas y están en todos los ambientes.

Estudiante 4. 2015-1. La estrategia de enseñanza es buena, a medida que se ve la teoría se complementa con los laboratorios y exposiciones de hongos de los compañeros, y así se entiende mucho más el tema.

Estudiante 5. 2015-2. La observación de los hongos permite el reconocimiento de sus características morfológicas. La investigación en hongos se profundiza con los laboratorios, seminarios y proyecto.

Estudiante 6. 2016-1. Las prácticas de laboratorio, los seminarios y proyecto facilitan la apropiación del conocimiento de biología de hongos y motivan el trabajo en equipo.

Estudiante 7. 2016-2. En el curso se conoce el mundo tan diverso de los hongos, vemos su reproducción, ecología, aplicación industrial, nutrición, metabolismo y con este aprendizaje podemos llegar a los estudiantes de colegio en la realización de las prácticas pedagógicas.

Estudiante 8. 2016 -2. Me parece un espacio académico que ha facilitado mi proceso de licenciada en formación, ya que las temáticas y estrategias del curso y laboratorios generan experiencias en el estudio de los hongos.

Estudiante 9. 2017-2. El desarrollo del curso es muy indispensable para la formación del Licenciado en Biología porque nos permite entender mejor la importancia de los hongos a nivel investigativo y educativo. También se relaciona con biotecnología, ecología y medicina.

Estudiante 10. 2017-2. Me gustó mucho desarrollo del proyecto de investigación ya que podemos identificar en campo macrohongos y su reconocimiento nos permite entender cómo se encuentran en el ecosistema y sus relaciones.

\section{Referencias}

Alexopoulus, C. J. (1996). Introductory Mycology (4th edition). John Willey and Sons. INC.

Angarita, J. (2011). Diseño de una estrategia pedagógica para la enseñanza de la biología de los organismos, a través de las quecas (Scaptocoris sp., Cydnidae) (Tesis de Maestría). Universidad Nacional de Colombia. Sede Bogotá.

Balvanera, P. (1995). La enseñanza de las ciencias biológicas. Perfiles Educativos, 68. Instituto de Investigaciones sobre la Universidad y la Educación. México.

Berrin, J. G., Navarro, D., Couturier, M., Oliver., Grilse., Hahn., ... y Lesage-Meessen L. (2012). Exploring the natural fungal biodiversity of tropical and temperate forests toward improvement of biomass conversion. Applied and Environmental Microbiology, 78 (18), 6483-6490.

Blackwell, M., Hibbett, D. S., Taylor, J. W. y Spatafora, J. W. (2007) [2006]. Research coordination networks: a phylogeny of kingdom Fungi. Mycologia, 98, 829-837.

Hibbett, D., Binder, M., Bischoff, J., Blackwell, M., Cannon, P., Eriksson, O., ... y Zhang, N. (2007). A higher-level phylogenetic classification of the Fungi. Mycological Research, 111 (5), 509-547.

Jiménez, H. M. (2018). Syllabus Seminario Biología de Hongos [Documento]. Universidad Pedagógica Nacional. Departamento de Biología.

Jiménez, H. M. (2012). Proyectos de Investigación en el Aula, una propuesta para la formación integral en la Escuela de Cadetes de Policía "General Francisco de Paula Santander". Revista ECSAN, 136, 16-19. ISSN 0120 - 582-X

Keeling, P. J. y Fast, N. M. (2002). Microsporidia: biology and evolution of highly reduced intracellular parasites. Annual Review of Microbiology, 53, 96-115.

Pantoja, J. C. y Covarrubias, P. (2013). La enseñanza de la biología en el bachillerato a partir del aprendizaje basado en problemas (ABP). Perfiles Educativos, 35 (139), 93-109. IISUE - UNAM, México. 